in BE (high grade dysplasia (HGD)/intramucosal adenocarcinoma) and patients with non-dysplastic BE (NDBE). Endoscopic resection margins/targeted biopsy site histology served as the ground truth for dysplasia in videos. Videos were annotated for definite visual presence of dysplasia. We trained a convolutional neural network with a Resnet101 architecture to classify video frames into dysplastic or non-dysplastic using randomly selected frames from annotated videos.

Results 58 patients each with high quality video frames of magnification areas of BE (34 dysplasia, 24 NDBE) were included. Performance was evaluated using a 15 -fold cross validation methodology. 76,496 (47,438 dysplasia, 29,058 NDBE) magnification video frames were analysed by the neural network. All dysplastic and non-dysplastic frames were included.

We used an exponentially weighted moving average of consecutive frames to make a diagnosis of dysplasia. The network achieved a per frame sensitivity of $82 \%$, specificity of $82 \%$, and Area under the ROC of 90\%. The mean assessment speed per frame was 0.0135 seconds (standard deviation, + 0.006) (Figure 1).

Conclusion The neural network can characterise BE dysplasia with high accuracy and speed on magnification endoscopic images. Whole video frames were used to train and test the

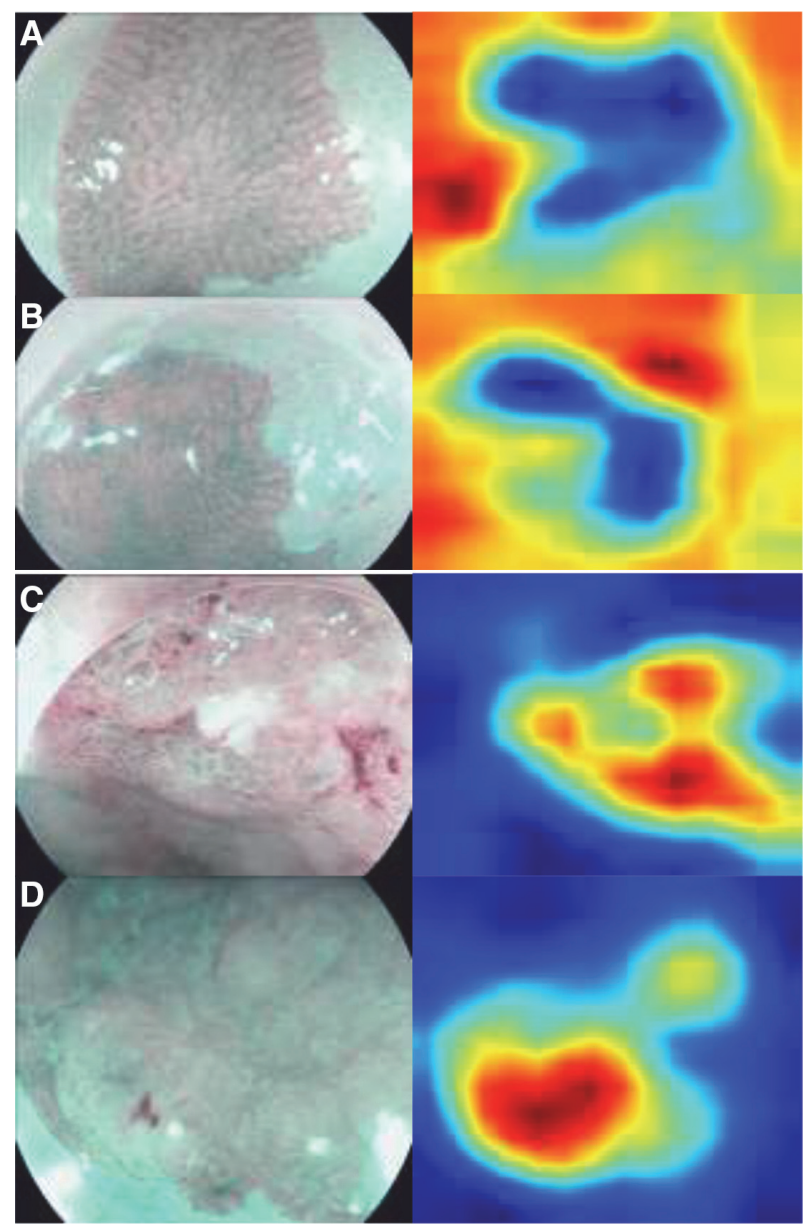

Abstract HFR-2 Figure 1 Areas of normal $B E(A, B)$, dysplastic $B E$ $(C, D)$ with corresponding heatmap with correct predictions generated by the network. The network assesses the blue area when making a negative diagnosis $(A, B)$ and the red area when making a positive diagnosis $(C, D)$ data moving it towards real time automated diagnosis. This will potentially aid endoscopists to make key decisions regarding endoscopic sampling and resection in BE during the same endoscopic session.

\section{HFR-3 PATIENT REPORTED OUTCOMES ON SYMPTOM IMPROVEMENT- A GOOD PREDICTOR OF HISTOLOGICAL REMISSION IN EOSINOPHILIC ESOPHAGITIS?}

${ }^{1}$ Pavit Luthra*, ${ }^{2}$ Konstantinos Giaslakiotis, 'Sami Hoque. 'Department of Gastroenterology, Barts Health NHS Trust, London, UK; ${ }^{2}$ Department of Pathology, Barts Health NHS Trust, London, UK

\subsection{6/gutjnl-2021-BSG.62}

Introduction Eosinophilic esophagitis (EoE) is a chronic immune-mediated inflammatory condition of the oesophagus. Randomized control trials frequently use histological remission as a definition of treatment effect, although in clinical practice there is often de-escalation of therapy once there is patient reported improvement in symptoms. However, persistent mucosal inflammation still risks tissue re-modelling and may lead to fibrosis and stricture formation. We aim to assess if patient reported outcomes (PRO) of symptom improvement were a good predictor of histological remission.

Methods We performed a retrospective observational analysis and identified 30 patients with EoE at Barts Health NHS trust between 2016-2020. EoE was defined as symptoms of dysphagia and/or food bolus obstruction with eosinophil count $\geq 15$ per high power field in proximal and distal oesophageal biopsies. All patients had subsequent follow up with clinical, endoscopic and histologic re-assessment after a minimum eight week period of treatment. Dichotomous outcomes were compared between patient reported global improvement in symptoms (improvement or no improvement) and histological remission (eosinophil count $<15$ eos/hpf).

Results 19/30 (63.33\%) patients were male. The median age was 35.9 years (age range 23-56). 18/30 (60\%) patients had atopic disorders whilst $8 / 30(26.67 \%)$ had $\operatorname{IgE}$ mediated food allergies. 22/30 (73.33\%) received PPI as first line therapy, 6/ $30(20 \%)$ received topical steroids and $2 / 30$ (6.67\%) had combined therapy with PPI and inhaled fluticasone. All patients had a minimum of 8 weeks treatment prior to reassessment. The mean duration of therapy was 38 weeks $(+/-$ 24.28). There was no association between PRO of global symptom improvement and histological remission across all treatment groups $(p=0.55)$. There was a mean increase of $10.33 \mathrm{eos} / \mathrm{hpf}$ on repeat biopsies. When specifically assessing outcomes following PPI therapy; 18/22 (81.8\%) reported improvement in symptoms, however only 4/22 (18.2\%) achieved histological remission $(\mathrm{p}=0.55)$.

Conclusions Microscopic disease activity can persist despite patient reported symptom improvement. Hence histology is the ideal marker of inflammation for disease activity. Our findings support European guidelines reinforcing that targeting mucosal healing should be the goal of therapy. Treatment should not de-escalated if there is ongoing mucosal inflammation. Standardised definitions of histologic response and remission using validated scoring systems, as well as a biopsy procurement protocol are needed to further guide clinical practice and decision making. 